\title{
Análisis de las infecciones del orificio de salida del catéter peritoneal. Efectividad de un protocolo basado en la aplicación de mupirocina tópica diaria
}

\author{
Silvia González-Sanchidrián ${ }^{1}$, María del Carmen Nacarino-Muriel ${ }^{1}$, Ana María García-Girón ${ }^{1}$, Fidel \\ Fernández-Vivas ${ }^{1}$, María del Carmen Pazos-Pacheco을 Sandra Gallego-Domínguez ${ }^{1}$
}

${ }^{1}$ Servicio de Nefrología. Hospital San Pedro de Alcántara. Complejo Hospitalario Universitario de Cáceres. Cáceres. España

${ }^{2}$ Servicio de Microbiología Clínica. Hospital San Pedro de Alcántara. Complejo Hospitalario Universitario de Cáceres. Cáceres. España

Como citar este artículo:

González-Sanchidrián S, Nacarino-Muriel MC, García-Girón AM, Fernández-Vivas F, Pazos-Pacheco MC, Gallego-Domínguez S. Análisis de las infecciones del orificio de salida del catéter peritoneal. Efectividad de un protocolo basado en la aplicación de mupirocina topica diaria. Enferm Nefrol. 2021 Abr-Jun;24(2):163-73

\section{Resumen}

Objetivos: Analizar las infecciones del orificio de salida del catéter peritoneal, identificando los principales factores relacionados con las mismas; asimismo se ha comparado la efectividad de un protocolo basado en la utilización diaria de mupirocina tópica en la cura del orificio de salida frente a la utilización suero salino.

Material y Método: Estudio observacional, retrospectivo. Recogimos variables demográficas, clínicas, la etiología y evolución de los episodios de infección del orificio en un periodo de 2 años. Utilizamos un protocolo de profilaxis con suero fisiológico y con mupirocina crema al $2 \%$.

Resultados: Estudiamos 75 pacientes, edad media $60,3 \pm 13,9$ años, $66,7 \%$ varones, mediana de tiempo en diálisis peritoneal 24,3 meses (rango intercuartílico 11 48,7 ). La incidencia de infección del orificio de salida fue del $26,7 \%$, el $77 \%$ fueron originadas por microorganismos de piel y mucosas. La infección del orificio de salida se relacionó con el estado portador nasal de Staphylococcus aureus $(p=0,048)$ y la extrusión del dacron externo $(p=0,004)$. De los 4 pacientes que presentaron una peritonitis secundaria a la infección del orificio de salida, 3 fueron trasferidos a hemodiálisis $(p=0,025)$.

\section{Correspondencia:}

Silvia González Sanchidrián

E-mail: silvia_goz@hotmail.com
Con el protocolo de antibioterapia tópica diaria se redujo un $68 \%$ la tasa de infecciones.

Conclusiones: La exteriorización del anillo externo del catéter peritoneal es un factor predisponente de infecciones. La aplicación diaria de mupirocina es una estrategia efectiva para reducir la incidencia de infección del orificio y peritonitis. La erradicación de portadores nasales de Staphylococcus aureus puede contribuir a reducir la pérdida del catéter y la transferencia a hemodiálisis.

PALABRAS CLAVE: catéter peritoneal; diálisis peritoneal; infección del orificio de salida; mupirocina; peritonitis.

\section{Analysis of peritoneal catheter exit-site infections. Effectiveness of a protocol based on daily topical mupirocin application}

\section{Summary}

Objectives: To analyze the characteristics of catheter related exit-site infections, identifying the main related factors. Likewise, the effectiveness of a protocol based on the daily use of topical mupirocin in the exit site prophylaxis strategy has been compared with the use of saline solution. 
Material and Method: Observational retrospective study. Demographic and clinical variables, the etiology and evolution of the episodes of infection of the orifice in a period of 2 years were collected. A prophylaxis protocol with saline solution and $2 \%$ mupirocin cream was used.

Results: We recruited 75 patients, mean age $60.3 \pm 13.9$ years, $66.7 \%$ male, median time on peritoneal dialysis 24.3 months (interquartile range 11-48.7). The incidence of exit site infection was $26.7 \%, 77 \%$ were produced by skin and mucous microorganisms. The exit site infection was associated to Staphylococcus aureus nasal carriage $(p=0.048)$ and the superficial cuff extrusion $(p=0.004)$. 4 of the patients who presented peritonitis secondary to exit site infection, 3 were switch to hemodialysis $(p=0.025)$. Daily topical antibiotic therapy protocol reduced the exit site infection rate by $68 \%$.

Conclusions: The superficial cuff externalization is a predisposing factor for infections. Daily application of mupirocin is an effective strategy in reducing the incidence of exit site infections and peritonitis. The eradication of nasal carriers of Staphylococcus aureus can help reduce catheter loss and transfer to hemodialysis.

KEYWORDS: peritoneal catheter; peritoneal dialysis; exit-site infection; mupirocin; peritonitis.

\section{Introducción}

Entre el 5 y el $30 \%$ de los pacientes con insuficiencia renal cónica que precisan tratamiento renal sustitutivo lo hacen mediante diálisis peritoneal $(D P)^{1,2}$. El éxito de cualquier técnica de diálisis a largo plazo, se basa en disponer de un acceso seguro y duradero. En DP, un catéter peritoneal (CP) correctamente implantado, bien funcionante y sin complicaciones, contribuirá a conseguir un tratamiento dialítico de calidad y con unos resultados óptimos ${ }^{1}$.

EI CP es un dispositivo invasivo que puede servir de reservorio para las bacterias ${ }^{2}$, por lo que debe estar fabricado de un material biocompatible, duradero y resistente a la colonización bacteriana. Además, debe estar adecuadamente localizado, que altere mínimamente la pared abdominal, sin desplazamientos, y capaz de minimizar las complicaciones con unos cuidados sencillos ${ }^{3}$.
La infección del orifico de salida (IOS) es una complicación en los pacientes en DP, se asocia a un mayor riesgo de peritonitis y es una de las principales causas de retirada del $\mathrm{CP}^{4}$. Por ello, el cuidado del orificio de salida (OS) del CP y la implementación de estrategias encaminadas a la prevención de complicaciones asociadas al catéter y al orificio suponen una parte fundamental del buen desarrollo de la técnica de DP ${ }^{1}$.

Se han identificado varios factores predisponentes para la infección del CP: la presencia de una barrera fibrótica débil, una mala epitelización del oficio, la dirección no caudal de la salida del $C P$, el uso de catéter de un sólo manguito o los traumatismos locales. La falta de cuidados diarios del OS, factores sistémicos, ciertos agentes microbiológicos y los portadores de Staphylococcus aureus nasal también pueden contribuir a ello ${ }^{1-4}$.

Existe una gran variabilidad en los protocolos de cuidado del OS y las tasas de infección del catéter difieren de unos centros a otros. Un elevado porcentaje de IOS debe hacernos reconsiderar las estrategias para el manejo y reducción de complicaciones en DP5,6.

El posicionamiento de la Sociedad Internacional de Diálisis Peritoneal (International Society for Peritoneal Dialysis, ISPD) acerca de las infecciones relacionadas con el catéter, recomienda con un nivel de evidencia 1 y grado de recomendación $\mathrm{A}$, la aplicación tópica diaria de crema antibiótica en la salida del $C P^{4}$.

El objetivo de este trabajo ha sido analizar las IOS del catéter peritoneal, identificando los principales factores relacionados con las mismas; así mismo se ha comparado la efectividad de un protocolo basado en la utilización diaria de mupirocina tópica en la cura del OS, frente a la utilización de suero salino.

\section{Material y Método}

Se realizó un estudio observacional retrospectivo, en la unidad de DP del Complejo Hospitalario de Cáceres (CHUC), de un periodo de 2 años, desde el 1 de enero de 2017 al 31 de diciembre de 2018.

El Área de Salud de Cáceres da asistencia sanitaria directa a 196.363 habitantes (48\% de la población de la provincia de Cáceres) con 520 camas de hospitalización, distribuidas en dos hospitales. EI CHUC es la referencia en DP para la provincia con una media de 49,8 pacientes en programa en los últimos 5 años. 
Los criterios de inclusión fueron: pacientes adultos tratados en la unidad de DP desde la implantación del CP, que hubieran dado su consentimiento para participar en el estudio y que completaran ambos protocolos de actuación (Anexo 1). Se excluyeron pacientes pediátricos.

Se recogieron variables sociodemográficas y clínicas: edad, sexo, modalidad de la técnica de DP y tiempo en la misma, complicaciones mecánicas, episodios de peritonitis, estado portador nasal de $S$. aureus, tratamiento dialítico previo, necesidad de DP asistida, salidas de programa y comorbilidades asociadas. En relación a las IOS, se han recogido los episodios de IOS, el germen causante, la evolución, así como la estacionalidad de las infecciones.

Se han analizado globalmente las causas relacionadas con IOS en el total de pacientes en el periodo 20172018, y se han comparado las infecciones entre el año 2017, donde la cura del OS se llevaba a cabo con suero fisiológico, y el año 2018, en el que se utilizaba mupirocina tópica al 2\%, (Anexo 1 ).

\section{Protocolo de definición, diagnóstico, tratamiento y actuación de la IOS, utilizada:}

- La infección del OS estaba definida por criterios clínicos, utilizando la clasificación de Twardowski. En todos los casos, se había recogido muestra para cultivo aerobio/anaerobio, mediante hisopo, ante la presencia de enrojecimiento y exudado purulento del OS. En el periodo correspondiente al año 2018, en el que se utilizó mupirocina en el cuidado del OS, la muestra de exudado se tomaba preferiblemente transcurridas al menos 24 horas desde la última aplicación de antibiótico; en caso de cultivo negativo o no concluyente se extraía una segunda muestra de exudado. El tratamiento antibiótico para la IOS se indicaba siguiendo las recomendaciones específicas de las guías internacionales de la ISPD 4 .

- Los tratamientos se mantenían un mínimo de 2 semanas con antibioterapia basada en el resultado del antibiograma, salvo para los casos de Pseudomona spp que se cumplimentaron al menos 3 semanas. El tratamiento antibiótico no se discontinuaba en ningún caso hasta que el OS adquiría la valoración de buen estado o perfecto ${ }^{4}$.

- El proceso infeccioso se consideraba curado cuando tras finalizar el tratamiento antibiótico desaparecían los signos de infección.
- Finalizado el tratamiento antibiótico, únicamente se realizaba un exudado de control en un intervalo de tiempo de $7 \pm 2$ días en aquellos 0 S equívocos o con algún signo de inflamación y/o infección. Se consideraba recidiva la IOS por el mismo microorganismo o con cultivo negativo en el primer mes de haber completado el tratamiento antibiótico, y reinfección cuando el episodio se producía por un agente distinto a la primoinfección. Cuando fue necesario se utilizó el apoyo de la ultrasonografía para el diagnóstico de la IOS y la evaluación de la respuesta al tratamiento ${ }^{4}$.

El ratio de IOS se definió como la incidencia anual de IOS del programa en función del número de pacientes y el tiempo de exposición ${ }^{5}$. Todos los CP de los pacientes analizados eran de tipo Tenckhoff con plomada, de 2 anillos y fueron colocados mediante laparoscopia.

Para el tratamiento de los datos se utilizó el software estadístico SPSS (SPSS v. 15.0, SPSS Inc., EE. UU.). Para el análisis descriptivo se utilizó la media y la desviación estándar para las variables cuantitativas, y la frecuencia y los porcentajes para las cualitativas. La comparación entre grupos se realizó mediante el test $\mathrm{t}$ de Student para las variables cuantitativas y el chi Cuadrado o test de Fisher para las cualitativas. Las variables estadísticamente significativas en el análisis univariante fueron incluidas en un análisis multivariante utilizando el modelo de regresión logística con el método Wald. Un valor de $p<0,05$ se consideró estadísticamente significativo.

Los datos se obtuvieron de la historia clínica y de la base de datos del registro de actividad de nuestra unidad. No se han incluido datos de carácter personal que pudieran identificar a los pacientes de forma directa o indirecta. El estudio se llevó a cabo cumpliendo la Ley orgánica 3/2018, de 5 de diciembre de protección de datos personales y garantía de los derechos digitales.

\section{Resultados}

En el periodo 2017-2018, estuvieron incluidos en el programa de DP de nuestra Unidad 75 pacientes, los cuales cumplieron los criterios de inclusión en este estudio retrospectivo.

La edad media de estos pacientes fue de $60,3 \pm 13,9$ años, 50 hombres $(66,7 \%)$ y 19 diabéticos $(25,3 \%)$. El $45 \%(n=34)$ de los pacientes tenían registrado un índice de Charlson $\geq 5$ puntos y un $9 \%(n=7)$ eran inmu- 
Etiología de la enfermedad renal, n(\%)

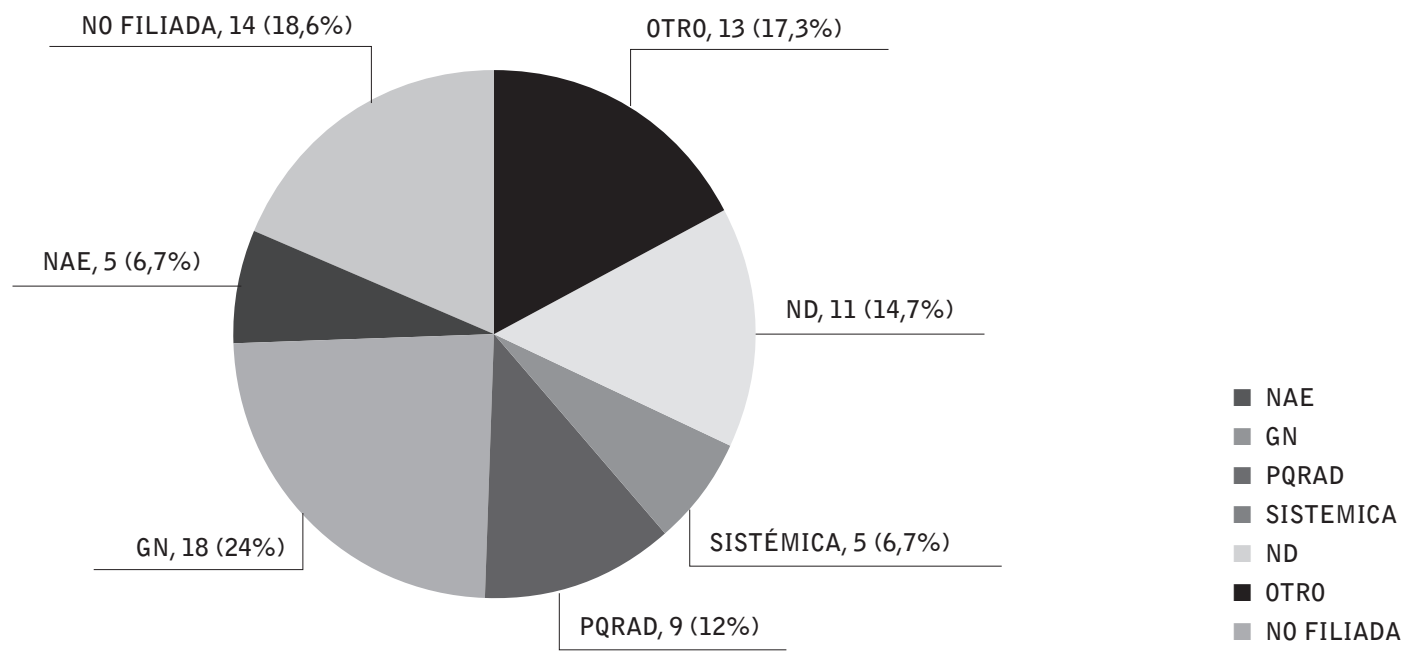

Figura 1. Distribución de la etiología de la enfermedad renal en la muestra de pacientes.

NAE: Nefroangioesclerosis; GN: Glomerular; PQRAD: Poliquistosis Renal Autosómica Dominante; ND: Nefropatía Diabética.

nodeprimidos, considerándose así por su tratamiento o enfermedad de base.

La etiología de la enfermedad renal más prevalente fue la glomerular, seguida de la patología no filiada (Figura 1). Un alto porcentaje de pacientes procedían de la consulta de enfermedad renal crónica avanzada (ERCA). La técnica mayoritaria era la diálisis peritoneal automatizada (DPA) sobre la diálisis peritoneal continua ambulatoria (DPCA), con una mediana de tiempo en DP de 24,3 meses (rango intercuartílico de 11 a 48,7 meses) (Tabla 1). Las salidas de programa fueron mayoritariamente por trasplante renal. De los 16 pacientes que fueron transferidos a hemodiálisis (el $21,3 \%$ de las salidas), la IOS se relacionó con la salida de programa por peritonitis $(p=0,025)$. No encontramos diferencias estadísticamente significativas con las demás causas de abandono de la técnica (Tabla 1). A dos pacientes se les retiró el CP por disfunción sin haber podido iniciar la técnica dialítica.

En el periodo de estudio registramos un total de 48 episodios de IOS en 20 pacientes, lo que supone una incidencia del $26,7 \%$. La IOS fue más frecuente en varones. De los 48 episodios de IOS, el $4 \%(n=2)$ fueron polimicrobianas y el $77,1 \%(n=37)$ fueron originadas por microorganismos de piel, mucosas y orofaringe. En 32 episodios $(66,7 \%)$ el agente causal fue un microorganismo grampositivo, en $14(29,2 \%)$ un gramnegativo y en $2(4,2 \%)$ un hongo; 12 episodios $(25 \%)$ fueron debidos a $S$. aureus y $7(14,6 \%)$ a Staphylococcus spp. coagulasa negativos, sólo tuvimos un caso por Pseudomona aeruginosa $(2,1 \%)$. No tuvimos ningún caso de IOS por criterios clínicos con cultivo negativo. El porcentaje de curación fue del 69\% (33 episodios) con un único ciclo antibiótico, el 31\% restante (15 episodios) precisó de más de un ciclo de tratamiento y 2 pacientes precisaron recambio del CP. De los 20 pacientes con IOS, 7 (35\%) presentaron una peritonitis secundaria a IOS. Los datos comparativos más relevantes de 2017 y 2018 se muestran en la Tabla 2. En la Tabla 3 se muestra una descripción de la etiología de las IOS, el hábitat habitual del germen causal y el número de episodios registrados por cada etiología en el periodo global de estudio y, diferenciadamente, antes y después de la aplicación del nuevo protocolo para el cuidado del OS con mupirocina.

De los 75 sujetos a estudio, 15 (20\%) tenían frotis nasal positivo para $S$. aureus, de los cuales cerca de la mitad ( $n=7,46,7 \%)$ desarrolló una complicación infecciosa del OS. No hubo diferencias entre las estaciones del año. El 34,6\% (26 pacientes) tenía una exteriorización del anillo externo del CP, bien espontánea o bien realizada mediante técnica de Friedrich. En la Tabla 1 se recogen las características de los pacientes que presentaron IOS frente a los que quedaron libres de dicha complicación. No hubo ningún caso de exitus derivado de la IOS o a causa de una peritonitis secundaria a este motivo. 
Tabla 1. Principales variables demográficas y factores de riesgo estudiados entre los pacientes con infección del orificio de salida (IOS) y $\sin$ IOS.

\begin{tabular}{|c|c|c|c|c|}
\hline Variables & $\begin{array}{c}\text { Total, n (\%) } \\
(n=75)\end{array}$ & $\begin{array}{c}\text { IOS, } n(\%) \\
(n=20)\end{array}$ & $\begin{array}{c}\text { No IOS, } n(\%) \\
(n=55)\end{array}$ & $\mathbf{p}$ \\
\hline Edad (años) & $60,3 \pm 13,9$ & $59,8 \pm 13,7$ & $60,4 \pm 14,1$ & 0,838 \\
\hline Género (hombres) & $50(66,7 \%)$ & $15(75 \%)$ & $35(63,6 \%)$ & 0,356 \\
\hline Índice de Charlson & $4,6 \pm 2,3$ & $4,8 \pm 1,9$ & $4,9 \pm 2,4$ & 0,814 \\
\hline $\mathrm{DM}$ & $19(25,3 \%)$ & $6(30 \%)$ & $13(23,6 \%)$ & 0,575 \\
\hline Inmunodepresión & $8(10,7)$ & $1(5 \%)$ & $7(12,7 \%)$ & 0,338 \\
\hline Procedencia ERCA & $67(89,3 \%)$ & $17(85 \%)$ & $50(90,9 \%)$ & 0,244 \\
\hline Tiempo en DP (meses) & $34,3 \pm 24,6$ & $32,1 \pm 27,4$ & $36,3 \pm 36,4$ & 0,638 \\
\hline Modalidad DPCA & $23(30,66 \%)$ & $5(25 \%)$ & $18(32,7 \%)$ & 0,521 \\
\hline Modalidad DPA & $52(69,3 \%)$ & $15(75 \%)$ & $37(67,8 \%)$ & 0,521 \\
\hline DP asistida & $11(14,6 \%)$ & $1(5 \%)$ & $10(18,2 \%)$ & 0,154 \\
\hline Portador S. aureus nasal & $15(20 \%)$ & $7(35 \%)$ & $8(14,5 \%)$ & 0,048 \\
\hline Extrusión cuff externo CP & $26(34,6 \%)$ & $12(60 \%)$ & $14(25,5 \%)$ & 0,003 \\
\hline Recambio CP & $7(9,3 \%)$ & $2(10 \%)$ & $5(9,1 \%)$ & 0,905 \\
\hline Peritonitis & $22(29,3 \%)$ & $7(35 \%)$ & $15(27,3 \%)$ & 0,516 \\
\hline Salida de programa & $42(56 \%)$ & $12(60 \%)$ & $30(54,5 \%)$ & 0,674 \\
\hline -Exitus & $6(8 \%)$ & $2(10 \%)$ & $4(7,3 \%)$ & 0,780 \\
\hline -Trasplante renal & $17(22,7 \%)$ & $3(15 \%)$ & $14(25,5 \%)$ & 0,196 \\
\hline -Recuperación función renal & $1(1,3 \%)$ & $0(0 \%)$ & $1(1,8 \%)$ & 0,522 \\
\hline -Pérdida de seguimiento & $2(2,7 \%)$ & $0(0 \%)$ & $2(3,6 \%)$ & 0,359 \\
\hline -Trasferencia a HD & $16(21,3 \%)$ & $7(35 \%)$ & $9(16,3 \%)$ & 0,088 \\
\hline --Por peritonitis & $4(5,3 \%)$ & $3(15 \%)$ & $1(1,8 \%)$ & 0,025 \\
\hline --Por disfunción del CP & $5(6,7 \%)$ & $2(10 \%)$ & $1(5,5 \%)$ & 0,485 \\
\hline --Por infradiálisis & $4(5,3 \%)$ & $1(5 \%)$ & $3(5,5 \%)$ & 0,938 \\
\hline --Por deseo del paciente & $3(4 \%)$ & $1(5 \%)$ & $2(3,6 \%)$ & 0,790 \\
\hline
\end{tabular}

CP: Catéter Peritoneal; DM: Diabetes Mellitus; DP: Diálisis Peritoneal; DPA: Diálisis Peritoneal Automatizada; DPCA: Diálisis Peritoneal Continua Ambulatoria; ERCA: Enfermedad Renal Crónica Avanzada; HD: Hemodiálisis; IC: Intervalo de Confianza; IOS: Infección del Orificio de Salida.

Tabla 2. Comparación entre la frecuencia de IOS en el año 2017 y en el 2018 con dos protocolos distintos para la cura del 0S.

\begin{tabular}{lcc}
\hline Variables & 2017 & 2018 \\
\hline Protocolo de cura del OS & Suero fisiológico $0,9 \%$ & Mupirocina crema $2 \%$ \\
Total pacientes tratados (n) & 59 & 58 \\
Exposición a riesgo (meses) & 543,6 & 503,4 \\
Episodios de IOS (n) & 37 & 11 \\
Pacientes con IOS (n) & 18 & 2 \\
Incidencia IOS & $30,5 \%$ & $3,4 \%$ \\
Reinfección & $8 \%$ & $82 \%$ \\
Tasa de IOS & 0,82 episodios/paciente-año & 0,26 episodios/paciente-año \\
& 1,63 episodios cada 24 pacientes-meses & 0,52 episodios cada 24 pacientes-meses \\
Etiología & $18(48,6 \%)$ & $2(18,2 \%)$ \\
Cocos grampositivos & $7(18,9 \%)$ & $5(45,5 \%)$ \\
Bacilos grampositivos & $10(27 \%)$ & $4(36,3 \%)$ \\
Bacilos gramnegativos & $2(5,5 \%)$ & 0 (0\%)
\end{tabular}

IOS: Infección del Orificio de Salida; OS: Orificio de Salida. 
Tabla 3. Etiología de los episodios de infección del orificio de salida (IOS) en una cohorte de 48 episodios.

\begin{tabular}{|c|c|c|c|c|}
\hline Microorganismo & Hábitat & $\begin{array}{c}\text { Total } \\
(n=48, \%)\end{array}$ & $\begin{array}{c}2017 \\
(n=37, \%)\end{array}$ & $\begin{array}{l}2018 \\
(n=11, \%)\end{array}$ \\
\hline \multicolumn{5}{|l|}{ Cocos grampositivos } \\
\hline $\begin{array}{l}\text { Staphylococcus aureus } \\
\text { Staphylococcus capitis } \\
\text { Staphylococcus epidermidis } \\
\text { Staphylococcus hominis } \\
\text { Staphylococcus lugdunensis } \\
\text { Streptococcus viridans }\end{array}$ & $\begin{array}{l}\text { Piel, pelo, orofaringe, fosas nasales } \\
\text { Piel y mucosas } \\
\text { Piel y mucosas } \\
\text { Piel y mucosas } \\
\text { Piel y mucosas } \\
\text { Piel y mucosas }\end{array}$ & $\begin{array}{l}12(25 \%) \\
1(2,1 \%) \\
2(4,2 \%) \\
1(2,1 \%) \\
3(6,3 \%) \\
1(2,1 \%)\end{array}$ & $\begin{array}{c}11(29,7 \%) \\
1(2,7 \%) \\
1(2,7 \%) \\
1(2,7 \%) \\
3(8,1 \%) \\
1(2,7 \%)\end{array}$ & $\begin{array}{l}1(9 \%) \\
1(9 \%)\end{array}$ \\
\hline \multicolumn{5}{|l|}{ Bacilos grampositivos } \\
\hline $\begin{array}{l}\text { Actinobaculum massiliense } \\
\text { Bacillus vallismortis } \\
\text { Bacilus sp } \\
\text { Corynebacterium aurimucosum } \\
\text { Corynebacterium jeikeium } \\
\text { Corynebacterium macginleyi } \\
\text { Corynebacterium sp } \\
\text { Corynebacterium striatum } \\
\text { Corynebacterium xerosis } \\
\text { Corynebacteriun accolens } \\
\text { Stenotrophomonas maltophilia }\end{array}$ & $\begin{array}{l}\text { Piel, orina } \\
\text { Suelo, agua } \\
\text { Suelo, agua } \\
\text { Piel y mucosas } \\
\text { Piel y mucosas } \\
\text { Piel y mucosas } \\
\text { Piel y mucosas } \\
\text { Piel y mucosas } \\
\text { Piel y mucosas } \\
\text { Piel y mucosas } \\
\text { Suelo, ambientes húmedos }\end{array}$ & $\begin{array}{l}1(2,1 \%) \\
1(2,1 \%) \\
1(2,1 \%) \\
1(2,1 \%) \\
1(2,1 \%) \\
1(2,1 \%) \\
1(2,1 \%) \\
1(2,1 \%) \\
2(4,2 \%) \\
1(2,1 \%) \\
1(2,1 \%)\end{array}$ & $\begin{array}{l}1(2,7 \%) \\
1(2,7 \%) \\
1(2,7 \%) \\
1(2,7 \%) \\
2(5,4 \%) \\
1(2,7 \%)\end{array}$ & $\begin{array}{l}1(9 \%) \\
1(9 \%) \\
1(9 \%) \\
1(9 \%) \\
1(9 \%)\end{array}$ \\
\hline \multicolumn{5}{|l|}{ Bacilos gramnegativos } \\
\hline $\begin{array}{l}\text { Citrobacter amalonaticus } \\
\text { Fusobacterium nucleatum } \\
\text { Klebsiella oxytoca } \\
\text { Klebsiella pneumoniae } \\
\text { Ochrobactrum anthropi } \\
\text { Proteus mirabilis } \\
\text { Pseudomonas aeruginosa } \\
\text { Sphingomona paucimobilis }\end{array}$ & $\begin{array}{l}\text { Tracto GI } \\
\text { Tubo digestivo (cavidad oral) y vagina } \\
\text { Tracto GI y flora comensal de las mucosas } \\
\text { Tracto GI y flora comensal de las mucosas } \\
\text { Suelo, agua } \\
\text { Tracto GI, suelo } \\
\text { Suelo, agua } \\
\text { Suelo, agua }\end{array}$ & $\begin{array}{l}2(4,2 \%) \\
1(2,1 \%) \\
4(8,3 \%) \\
3(6,3 \%) \\
1(2,1 \%) \\
1(2,1 \%) \\
1(2,1 \%) \\
1(2,1 \%)\end{array}$ & $\begin{array}{l}1(2,7 \%) \\
4(10,8 \%) \\
3(8,1 \%) \\
1(2,7 \%) \\
1(2,7 \%)\end{array}$ & $1(9 \%)$ \\
\hline \multicolumn{5}{|l|}{ Otros } \\
\hline $\begin{array}{l}\text { Candida parapsilosis } \\
\text { Cryptococcus albidus }\end{array}$ & $\begin{array}{l}\text { Piel y mucosas } \\
\text { Excrementos de aves }\end{array}$ & $\begin{array}{l}1(2,1 \%) \\
1(2,1 \%)\end{array}$ & $\begin{array}{l}1(2,7 \%) \\
1(2,7 \%)\end{array}$ & \\
\hline
\end{tabular}

GI: Tracto Gastrointestinal; IOS: Infección del Orificio de Salida.

Tabla 4. Análisis multivariante de los factores asociados a la infección del orificio de salida (IOS).

\begin{tabular}{l|c|c|}
\hline Variables & $\mathbf{P}$ & OR (IC, 95\%) \\
\hline Portador S. aureus nasal & 0,175 & $3,384(0,390-3,764)$ \\
Extrusión cuff externo CP & $\mathbf{0 , 0 0 4}$ & $\mathbf{5 , 0 2}(\mathbf{1 , 6 5 - 1 5 , 2 7 )}$ \\
Trasferencia a HD por peritonitis & 0,058 & $7,105(0,684-9,076)$ \\
\hline
\end{tabular}

CP: Catéter Peritoneal; HD: Hemodiálisis.

En el análisis univariante, se relacionó con una mayor incidencia de IOS: ser portador nasal de $S$. aureus $(p=0,048)$ y la extrusión del dacron externo $(p=0,003)$. La IOS condicionó, además, el abandono de la técnica y la trasferencia a hemodiálisis por peritonitis en 3 de los 20 pacientes ( $15 \%$ ) que habían presentado esta complicación del OS $(p=0,025)$. En el modelo de regresión logística del análisis multivariante la IOS únicamente se asoció a la exteriorización del cuff externo (Tabla 4).

Con el protocolo de antibioterapia tópica diaria se redujo un $68 \%$ la tasa de IOS, que pasó de 0,82 a 0,26 episodios/paciente-año. Las IOS por S. aureus se redujeron en más del $90 \%$ con el protocolo basado en mupirocina tópica. La tasa de peritonitis anual también se redujo, de 0,82 episodios de peritonitis por 
paciente/año en 2017 frente a 0,45 en 2018. No hemos evidenciado resistencias a antibióticos en el periodo estudiado.

\section{Discusión}

El plan de calidad científico-técnica y de mejora continua de calidad en DP recomienda un seguimiento periódico de varios indicadores que, a modo de herramienta de uso interno, nos permite identificar problemáticas o verificar el cumplimiento de objetivos previamente definidos, con la finalidad de mejorar los resultados de nuestra práctica clínica5.

Gracias a este proceso de evaluación, en 2017 detectamos un aumento en la tasa de IOS de nuestro programa. Esto nos movió a plantearnos un cambio en la estrategia que seguíamos para los cuidados del OS, realizada con suero fisiológico en la cura diaria del orificio, que reemplazamos por la aplicación de mupirocina tópica diaria al $2 \%$ en el seno del $0 \mathrm{~S}$, según la recomendación de las guías internacionales de DP4.

Del análisis de este estudio hemos podido observar que tenemos una tasa de IOS en consonancia con las registradas por otros centros con la aplicación de mupirocina diaria (las tasas de infección del catéter descritas varían entre 0,05 y 1,02 episodios/paciente-año) ${ }^{5}$. En los pacientes inmunodeprimidos y los que precisan DP asistida se registró un menor número de IOS; probablemente en estos pacientes se incida más en la importancia del autocuidado y en la prevención de complicaciones infecciosas. En nuestro trabajo no hemos encontrado diferencias en la aparición de episodios de IOS respecto a la edad, el género, la modalidad de tratamiento, el tiempo en la técnica o las comorbilidades asociadas. Cuando se diagnosticó una IOS la pauta de tratamiento antibiótico se realizó en base al antibiograma y de acuerdo a las recomendaciones de las guías de la ISPD $^{4,7,8}$. Dado que el valor predictivo de un cultivo positivo sin clínica acompañante, especialmente por gramnegativos es bajo, decidimos no obtener cultivos de control en todos los casos después del tratamiento 9 . El cultivo del OS se limitó sólo a los casos en los que persistieron signos de infección después de haber finalizado la pauta antibiótica dirigida.

La IOS fue fundamentalmente causada por gérmenes de piel y mucosas; hallazgo que podría estar en relación con la alta tasa de portadores de $S$. aureus nasal de nuestra unidad. A modo de curiosidad, tuvimos un caso por Cryptococcus albidus, un género de hongo cuyo reservorio habitualmente se encuentra en las aves, y muy frecuentemente en la palomas, en una paciente del entorno rural ${ }^{7,8}$. C. albidus es un germen inusual en pacientes de DP, pero ha sido descrito excepcionalmente en peritonitis. Ragupathi y cols. reportan el primer caso de peritonitis por $C$. albicus en una paciente inmunodeprimida que fue tratada de manera exitosa con anfotericina $\mathrm{B}^{10}$.

En nuestra unidad realizamos cultivos de muestras nasales cada 6 meses en todos los pacientes y cuidadores si la DP es asistida, para estudiar el estado de portador de $S$. aureus y aplicar tratamiento de erradicación si fuera necesario con mupirocina nasal ${ }^{4}$. Aunque existen varios regímenes de antibioterapia profiláctica para el cuidado del OS que han demostrado su utilidad ${ }^{4,11,12}$, por la alta tasa de portadores nasales de $S$. aureus en nuestra unidad, nos planteamos el uso de mupirocina tópica en crema, cuya efectividad ha sido avalada en múltiples estudios ${ }^{13-16}$ y que ha demostrado ser una herramienta costo-efectiva ${ }^{17}$. Si bien es cierto que, con el objetivo de evitar la generalización en el uso de antibióticos, podríamos habernos planteado el uso de mupirocina tópica exclusivamente en aquellos pacientes portadores de $S$. aureus $s^{4,12,14}$. Algunos estudios han documentado la aparición de resistencia a antibióticos con mupirocina en pautas de aplicación intermitente ${ }^{18-20}$, por lo que optamos por la aplicación diaria, que además refuerza el autocuidado del OS por el paciente y permite una consulta precoz en caso de sospecha de infección.

En nuestra experiencia, la IOS se relacionó con la exteriorización del cuff externo del CP, como única variable significativa en el análisis multivariante, un factor predisponente conocido de complicaciones infecciosas del $\mathrm{CP}^{1,21}$. La exteriorización espontánea es frecuente cuando el dacrón externo queda abocado muy próximo a piel y en ocasiones de realiza quirúrgicamente para retirar tejidos anormales o en casos de infección crónica ${ }^{22}$. Aunque la extrusión del dacrón puede realizarse con el objetivo de salvaguardar catéteres en algunos casos de IOS, no es una práctica habitual y debemos tener en cuenta que con dicho procedimiento estamos eliminando una barrera de protección frente a la infección $^{1,21-24}$. En nuestra unidad no es una práctica frecuente y se indica el recambio quirúrgico del CP en los casos de infección crónica del OS cuando el tratamiento antibiótico no es eficaz. Sólo en un paciente se exteriorizó el cuff quirúrgicamente antes del reemplazo del CP.

En la actualidad no hay suficientes datos para sugerir un objetivo mínimo para la tasa de IOS, y el indicador 
utilizado tendrá valor fundamentalmente como comparador dentro de la propia unidad. La incidencia de IOS y de peritonitis en nuestro estudio está dentro de los estándares establecidos, tanto nacionales como internacionales (menos de un episodio cada 24 pacientes-meses $)^{4,5}$. En nuestro estudio la tasa de IOS se redujo de 0,82 en 2017 a 0,26 episodios/paciente/año en 2018. No obstante, tal cual se indica en las guías de práctica clínica, deben ser parámetros constantemente sometidos a evaluación, revisables y mejorables, en todas las unidades de DP. Una observación importante ha sido que la IOS condicionó el abandono de la técnica de diálisis por peritonitis en un $75 \%$ de los casos. Aunque las pautas de la ISPD recomiendan el uso de antimicrobianos tópicos diarios en el OS para la prevención de peritonitis, su uso no es una práctica de rutina en todas las unidades ${ }^{25-27}$, principalmente por la aparición de resistencias a antibióticos que han causado una gran preocupación $n^{19,25,28}$. Por el contrario, sabemos que la peritonitis sigue siendo, dentro de las complicaciones infecciosas, el talón de Aquiles en los programas de DP por tanto, la implementación de medidas encaminadas a reducir la tasa de peritonitis contribuirá a mantener el éxito de nuestros programas ${ }^{1,5}$. Por ello, la individualización de protocolos en función de las características de cada centro y su entorno es fundamental para la elección de la estrategia más adecuada ${ }^{29}$.

Los cuidados del OS en los pacientes en programa de DP se realizan por el propio paciente. El entrenamiento en la técnica y la educación del paciente para su autocuidado es clave para prevenir complicaciones infeccio$\operatorname{sas}^{1,30}$.

Las principales limitaciones de este estudio han sido su carácter retrospectivo y unicéntrico, así como el limitado número de pacientes incluidos en el mismo. No se ha individualizado la colocación del CP en función de parámetros antropométricos, por lo que no se ha podido evaluar esta variable, aunque consideramos que una técnica quirúrgica individualizada y una correcta evaluación de la fisionomía del paciente antes de la implantación del catéter podría evitar la exteriorización del cuff que, a su vez, favorece la colonización bacteriana ${ }^{31}$.

En nuestro programa de diálisis mantenemos una constante actualización y revisión de los objetivos que marcan los planes de calidad, lo que nos ayuda a detectar problemas precozmente y mejorar resultados. La flexibilidad de nuestro programa para la demanda asistencial, permite una consulta precoz de los pacientes en caso de eventualidades. La IOS ha sido en todos los casos demostrada microbiológicamente, se han seguido las recomendaciones estandarizadas para su tratamiento y la detección de portadores de $S$. aureus está protocolizada en nuestro programa. La educación para el autocuidado en los pacientes en programas de DP juega un papel fundamental en la prevención de complicaciones infecciosas del acceso peritoneal, por lo que el equipo de enfermería de la unidad, experto y altamente cualificado participa en la educación y re-entrenamiento de los pacientes para unos adecuados cuidados del OS.

Estudios prospectivos, con un mayor número de casos y la experiencia acumulada de las unidades de DP, adaptadas a las peculiaridades de cada centro para la prevención de las complicaciones infecciosas, son necesarias para la obtención de buenos resultados en los programas de DP.

De nuestro análisis se deriva que, el estado portador de S. aureus nasal y, especialmente, la extrusión del anillo externo se asocia fuertemente con la IOS, determinando además el abandono de la técnica por peritonitis.

Los protocolos de cuidado del OS con antibióticos tópicos pueden ser efectivos; su instauración debe individualizarse y dependerá de la tasa de infecciones del OS en cada unidad de DP. Consideramos preciso insistir en la erradicación de portadores nasales de $S$. aureus para la prevención de las IOS.

\section{Agradecimientos}

A la Dra. Inés Castellano Cerviño por transmitirnos su pasión y su permanente búsqueda de la perfección en la diálisis domiciliaria. A Dña. Angelines Domínguez de la Calle, por su entrega y larga trayectoria dedicada a los pacientes renales. Al Dr. Pedro Jesús Labrador Gómez, por su colaboración en el análisis estadístico y la revisión crítica del artículo. A los Dres. Juan Ramón Gómez Martino Arroyo y Javier Deira Lorenzo por su inestimable apoyo constante a la investigación.

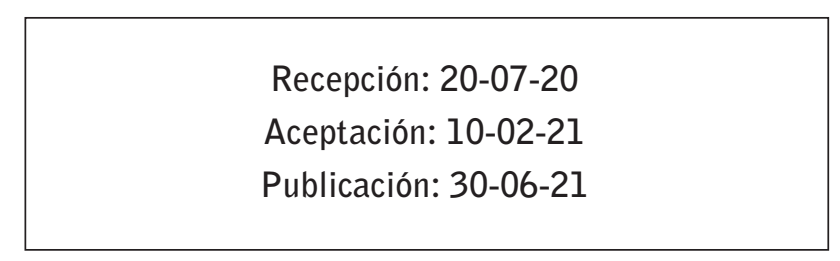




\section{Bibliografía}

1. Montenegro J, Correa R, Riella MC. Tratado de diálisis peritoneal. $2^{\mathrm{a}}$ ed. España: Elsevier; 2016.

2. Fariñas MC, García-Palomo JD, Gutiérrez-Cuadra M. Infecciones asociadas a los catéteres utilizados para la hemodiálisis y la diálisis peritoneal. Enferm Infecc Micribiol Clin 2008; 26(8):518-26.

3. Htay $H$, Johnson DW, Craig JC, Schena FP, Strippoli GF, Tong A et al. Catheter type, placement and insertion techniques for preventing catheter-related infections in chronic peritoneal dialysis patients. Cochrane Database Syst Rev 2019;31;5:CD004680.

4. Szeto CC, Li PK, Johnson DW, Bernardini J, Dong $J$, Figuereido $A E$ et al. ISPD catheter-related infection recomendations: 2017 update. Perit Dial Int 2017;37(2):141-54.

5. Bajo MA, Selgas R, Remón C, Arrieta J, ÁlvarezUde F, Arenas MD et al. Plan de calidad científicotécnica y de mejora continúa de calidad en diálisis peritoneal. Nefrología 2010;30(1):28-45.

6. Portolés J, Sánchez E, Janeiro D, Montenegro J. Peritonitis e infecciones del catéter en la diálisis peritoneal. En; Nefrología al día. Sociedad Española de Nefrología. 2019 [consultado 14 abr 2020]. Disponible en: https://www.nefrologiaaldia. org/es-articulo-peritonitis-e-infecciones-delcateter-223.

7. Murray PR, Rosenthal KS, Phaller MA. Medical Microbiology. $9^{\text {th }}$ ed. Philadelphia. Elsevier; 2020.

8. Bennett JE, Dollin R, Blaser MJ. Mandell, Douglas and Bennett's. Principles and Practice of Infectious Diseases. 9th ed. Philadelphia. Elsevier 2019.

9. Perez-Fontan M, Rodríguez Carmona A, Rosales M, García-Falcón T, Valdés F. Incidence and clinical significance of nasal and pericatheter colonization by gram-negative bacteria among patients undergoing chronic peritoneal dialysis. Nephrol Dial Transplant 2002;17:118-22.

10. Ragupathi L, Reyna M. Case Report of Cryptococcus Albidus Peritonitis in a Peritoneal Dialysis Patient and a Review of the Literature. Perit Dial Int 2015;35(4):421-7.

11. Strippoli GF, Tong A, Johnson D, Schena FP, Craig JC. Antimicrobial agents to prevent peritonitis in peritoneal dialysis: a systematic review of randomized controlled trials. Am J Kidney Dis 2004;44:591-603.

12. Strippoli GF, Tong A, Johnson D, Schena FP, Craig JC. Catheter-related interventions to prevent peritonitis in peritoneal dialysis: a systematic review of randomized controlled trials. J Am Soc Nephrol 2004;15:2735-46.

13. Tacconelli E, Carmeli Y, Aizer A, Ferreira G, Foreman MG, D'Agata EM. Mupirocin prophylaxis to prevent Staphylococcus aureus infection in patients undergoing dialysis: a meta-analysis. Clin Infect Dis 2003;37:1629-38.

14. Xu G, Tu W, Xu C. Mupirocin for preventing exitsite infection and peritonitis in patients undergoing peritoneal dialysis. Nephrol Dial Transplant 2010;25:587-92.

15. Lim CT, Wong KS, Foo MW. The impact of topical mupirocin on peritoneal dialysis infection in Singapore General Hospital. Nephrol Dial Transplant 2005;20:2202-6.

16. Davenport A. Do topical antibiotics reduce exit-site infection rates and peritonitis episodes in peritoneal dialysis patients? The Pan Thames Renal Audit. J Nephrol 2012;25:819-24.

17. Wong C, Luk IW, Ip M, You JH. Prevention of grampositive infections in peritoneal dialysis patients in Hong Kong: a cost-effectiveness analysis. Am J Infect Control 2014;42:412-6.

18. Cavdar C, Saglam F, Sifil A, et al. Effect of once-aweek vs thrice-a-week application of mupirocin on methicillin and mupirocin resistance in peritoneal dialysis patients: three years of experiencie. Ren Fail 2008;30:417-22.

19. Perez-Fontan M, Rosales M, Rodriguez-Carmona, García-Falcón T, Valdés F. Mupirocin resistance after long-term use for Staphylococcus aureus colonization in patients undergoing chronic peritoneal dialysis. Am J Kidney Dis 2002;39:337-41. 
20. Al-Hwiesh AK, Abdful-Rahman IS, Muhanna AI FA, et al. Prevention of peritoneal catheter infections in Saudi peritoneal dialysis: The emergence of high-level mupirocin resistance. Int $\mathrm{J}$ Artif Organs 2013;36:473-83.

21. Lin J, Ye H, Li J, Qiu Y, Wu H, Yi C, Lu S, et al. Prevalence and risk factors of exit-site infection in incident peritoneal dialysis patients. Perit Dial Int 2020;40(2):164-70.

22. Picó L, Picó L, Blaya $L$, Hernández MI, Serrano $\mathrm{RM}$, Lillo A. Infección del orificio de salida y anillo externo del catéter peritoneal tratada mediante técnica de Friedrich. Enferm Nefrol. 2013;16(Suppl1):S15-33

23. Kirmizis D, Bowes E, Ansari B, Cairns H. Exit-site recolocation: a novel, straightforward technique for exit-site infections. Perit Dial Int 2019;39(4):350-5.

24. Sun $M L$, Zhang $Y$, Wang $B$, Ma TA, Jiang $H$, Hu SL et al. Randomized controlled trials for comparison of laparoscopic versus conventional open catheter placement in peritoneal dialysis patients: a metaanalysis. BMC Nephrol 2020;21(1):60.

25. Nochaiwong S, Ruengorn C, Noppakun K, Panyathong S, Dandecha P, Sood MM et al. Comparative effectiveness of local application of chlorhexidine gluconate, muripocin ointment, and normal saline for the prevention of peritoneal dialyisis related-infections (COSMO-PD Trial): a multicenter randomized, double-blind, controlled protocol. Trials 2019;20(1):754.
26. Boudville N, Johnson DW, Zhao J, Bieber BA, Pisoni $\mathrm{RL}$, Piraino $B$ et al. Regional variation in the treatment and prevention of peritoneal dialysisrelated infections in the Peritoneal Dialysis Outcomes and Practice Patterns Study. Nephrol Dial Transplant. 2019;34(12):2118-26.

27. Lanot A, Bechade C, Verger C, Fabre E, Vernier I, Lobbedez T. Patterns of peritoneal dialysis catheter practices and technique failure in peritoneal dialysis: a nationwide cohort study. PLoS One. 2019;14(6):e0218677.

28. Annigeri R, Conly J, Vas S, Dedier H, Prakashan $\mathrm{KP}$, Bargman JM, et al. Emergence of mupirocinresistant Staphylococcus aureus in chronic peritoneal dialysis patients using mupirocin prophylaxis to prevent exit-site infection. Perit Dial Int. 2001;21(6):554-9.

29. Perl J, Fuller DS, Bieber BA, Boudville $N$, Kanjanabuch T, Ito $Y$, et al. Peritoneal dialysisrelated infection rates and outcomes: results from the peritoneal dialysis outcomes and practice patterns study (PDOPPS). Am J Kidney Dis 2020. pii: S0272-6386(19)31105-9.

30. Aguilera-Flórez AI, Sastre-López A, Linares-Fano B, Guerra-Ordoñez JR, Alonso-Rojo AC, PrietoVelasco M. Análisis del tiempo de entrenamiento y su relación con los episodios de peritonitis. Enferm Nefrol. 2020 Ene-Mar;23(1):54-9.

31. Crabtree JH, Shrestha BM, Chow KM, Figuereido $A E$, Povlsen JV, Wilkie $M$ et al. Creating and maintaining optimal peritoneal dialysis access in the adult patient: 2019 update. Perit Dial Int 2019;39(5):414-36. 


\section{Anexo 1.}

\section{A Protocolo para el cuidado del orificio de salida con suero fisiológico.}

- Utilizar una técnica aséptica (mascarilla y lavado de manos).

- Realizar los cuidados del OS todos los días.

- Los cuidados se deberán realizar coincidiendo con la ducha diaria. Se utilizará jabón líquido para su limpieza y se evitará el uso de agua no clorada en el lavado del orificio.

- Lavar cuidadosamente el orificio con la mano evitando esponjas o manoplas.

- Aplicar suero fisiológico sobre el OS y secar con gasas estériles no retirando costras insistentemente.

- Tapar con un apósito transpirable (opcional en orificios cicatrizados y sanos).

- Evitar traumas asegurando e inmovilizando firmemente el catéter.

- Evaluar en cada cura el estado del orificio para detectar alteraciones y/o signos de infección.

\section{B Protocolo para el cuidado del orificio de salida con mupirocina tópica en crema al $2 \%$.}

- Utilizar una técnica aséptica (mascarilla y lavado de manos).

- Realizar los cuidados del OS todos los días.

- Los cuidados se deberán realizar coincidiendo con la ducha diaria. Se utilizará jabón líquido para su limpieza y se evitará el uso de agua no clorada en el lavado del orificio.

- Lavar cuidadosamente el orificio con la mano evitando esponjas o manoplas.

- Aplicar suero fisiológico sobre el OS y secar con gasas estériles no retirando costras insistentemente.

- Aplicar una fina capa de muripocina en crema al 2\% de manera uniforme sobre el OS, con un suave masaje en movimientos circulares hasta su total absorción.

- Tapar con un apósito transpirable (opcional en orificios cicatrizados y sanos).

- Evitar traumas asegurando e inmovilizando firmemente el catéter.

- Evaluar en cada cura el estado del orificio para detectar alteraciones y/o signos de infección.

Este artículo se distribuye bajo una Licencia Creative Commons Atribución-NoComercial 4.0 Internacional. https://creativecommons.org/licenses/by-nc/4.0/

\section{Open Access (c) $\underset{\mathrm{EY}}{(\mathbf{\mathrm { NC }}}$}

